\title{
On The Existence of Levi Foliations
}

\author{
RENATA N. OSTWALD \\ Instituto de Matemática Pura e Aplicada - IMPA, Est. Dona Castorina 110 \\ 22460-320 Rio de Janeiro, Brasil
}

Manuscript received on September 20, 2000; accepted for publication on December 6, 2000;

presented by CÉSAR CAMACHO

\begin{abstract}
Let $L \subset \mathbb{C}^{2}$ be a real 3 dimensional analytic variety. For each regular point $p \in L$ there exists a unique complex line $l_{p}$ on the space tangent to $L$ at $p$. When the field of complex line

$$
p \mapsto l_{p}
$$

is completely integrable, we say that $L$ is Levi variety. More generally; let $L \subset M$ be a real subvariety in an holomorphic complex variety $M$. If there exists a real 2 dimensional integrable distribution on $L$ which is invariant by the holomorphic structure $J$ induced by $M$, we say that $L$ is a Levi variety. We shall prove:
\end{abstract}

Theorem. Let $\mathcal{L}$ be a Levi foliation and let $\mathcal{F}$ be the induced holomorphic foliation. Then, $\mathcal{F}$ admits a Liouvillian first integral.

In other words, if $\mathcal{L}$ is a 3 dimensional analytic foliation such that the induced complex distribution defines an holomorphic foliation $\mathcal{F}$; that is, if $\mathcal{L}$ is a Levi foliation; then $\mathcal{F}$ admits a Liouvillian first integral - a function which can be constructed by the composition of rational functions, exponentiation, integration, and algebraic functions (Singer 1992). For example, if $f$ is an holomorphic function and if $\theta$ is real a 1 -form on $\mathbb{R}^{2}$; then the pull-back of $\theta$ by $f$ defines a Levi foliation $\mathcal{L}: f^{*} \theta=0$ which is tangent to the holomorphic foliation $\mathcal{F}: d f=0$.

This problem was proposed by D. Cerveau in a meeting (see Fernandez 1997).

Key words: Levi foliations, holomorphic foliations, singularities, Levi varieties.

\section{ANNOUNCEMENT}

Let $\mathcal{L}$ be a Levi foliation and let $\mathcal{F}$ be the holomorphic foliation tangent to $\mathcal{L}$. Note that if $h$ in an holomorphic function such that $\mathcal{F}$ is $h$-invariant $\left(h^{*} \mathcal{F}=\mathcal{F}\right)$; then $\mathcal{L}$ is also $h$-invariant $\left(h^{*} \mathcal{L}=\mathcal{L}\right)$. We shall mainly use that property in order to prove

THEOREM. Let $\mathcal{L}$ be a Levi foliation and let $\mathcal{F}$ be the induced holomorphic foliation. Then $\mathcal{F}$ admits a Liouvillian first integral.

E-mail: ostwald@impa.br 
We proceed as follows:

We first show that if $\mathcal{L}$ is a Levi foliation, there exists analytic real functions $g_{1}, g_{2}$ such that: if $G=g_{1}+i g_{2}$, then the Levi foliation is defined by

$$
\mathcal{L}: \bar{G} \omega+G \bar{w}=0
$$

where $\omega$ is an holomorphic 1-form so that $\omega=0$ defines the holomorphic foliation $\mathcal{F}$ tangent to the Levi foliation $\mathcal{L}$. We then verify that; if $\mathcal{F}^{*}$ is the holomorphic foliation obtained from $\mathcal{F}$ after a finite number of blow-ups, there exists a Levi foliation $\mathcal{L}^{*}$ tangent to $\mathcal{F}^{*}$. Therefore, by Seidenberg Theorem (Seindenberg 1968), we analyse the foliation $\mathcal{F}^{*}$ for which all singularities are reduced.

Let $D$ denote the divisor obtained on the process of reducing the singularity and let $D_{j}$ denote the irreducible curves with normal crossings such that $D=\cup D_{j}$. We consider the induced Levi foliation on sections transversal to the holomorphic foliation through each component $D_{j}$ of the divisor. We show that the holomorphic diffeomorfisms for which the Levi foliation is invariant must satisfy an equation on one variable of the type

$$
h^{\prime}(z)=t \frac{F(h)}{F} ; \quad t \in \mathbb{R}
$$

We can then find an holomorphic coordinate system $y$ on the section such that

$$
F(y)=\frac{y^{k+1}}{1-\lambda y^{k}} .
$$

We refer to such coordinate system as a normalizable coordinate system. We verify that it is unique up to homographies.

If either $\lambda \neq 0$ or $k=0$, then $t=1$ for all solutions $h$ of the differential equation (*). Furthermore, if $k=0$, then the group of solutions of the differential equation is a linear group. On both cases we have an abelian group for the group of solutions of $(*)$. We can already conclude:

THEOREM A. Let $p$ be a singularity of the foliation

$$
\mathcal{F}: \omega=\lambda x d y+y d x+\{\text { higher order terms }\}=0 \quad \lambda \in \mathbb{R}^{*}-\mathbb{Q} .
$$

Suppose there exists a Levi foliation $\mathcal{L}$ tangent to $\mathcal{F}$. Then the singularity is analytically equivalent to a linear singularity.

Proof. For if there exists a Levi foliation, the holonomy associated to the singularity must satisfy an equation as $(*)$. If so, the order of $F$ at 0 cannot be but 1 ; that is, $\mathrm{k}=0$. The holonomy is linearizable; as a result, so is the singularity (Mattei \& Moussu 1980).

We still have to consider the case $\lambda=0$. There are solutions for which $t \neq 1,\left(h^{\prime}(0)\right)^{k}=\frac{1}{t} \in \mathbb{R}$. These solutions are necessarily linearizable, but not those for which $t=1$. The latter, though, also determine an abelian group. We shall then describe the abelian group of solutions of (*) for $t=1, k>0$. 
We can take an holomorphic coordinate system $(x, y)$ such that the group of solutions of the differential equation is in normalizable coordinate system on each transversal section $x=$ cte.

For an holomorphic vector field $X$, let $\exp X$ denote its exponential application, that is, its flow for $t=1$ :

$$
\exp \left(\xi(z) \frac{\partial}{\partial z}\right)(z)=z+f_{1}(z)+\frac{1}{2} f_{2}(z)+\frac{1}{3 !} f_{3}(z)+\ldots
$$

satisfying

$$
\left\{\begin{array}{l}
f_{1}=\xi \\
f_{n}=\xi f_{n-1}^{\prime}
\end{array}\right.
$$

If $h$ is a diffeomorfism which satisfies

$$
h^{\prime}(z)=\frac{h^{k+1}}{1-\lambda h^{k}} \frac{1-\lambda y^{k}}{y^{k+1}}
$$

then the $k$-th interate of $\mathrm{h} ; h^{k}$, is tangent to the identity. There exists $\mu$ such that $h^{k}$ is the exponential of the vector field:

$$
Y=2 \pi i \mu \frac{y^{k+1}}{1-\lambda y^{k}} \frac{\partial}{\partial y}
$$

that is

$$
h^{k}(w)=\exp \left(2 \pi i \mu \frac{y^{k+1}}{1-\lambda y^{k}} \frac{\partial}{\partial y}\right)(w) .
$$

Consequently

$$
h(w)=\exp \left(2 \pi i \frac{\mu}{k} \frac{y^{k+1}}{1-\lambda y^{k}} \frac{\partial}{\partial y}\right)(\epsilon w) ; \epsilon^{k}=1 .
$$

If

$$
X=x \frac{\partial}{\partial x}+y f(x, y) \frac{\partial}{\partial y}
$$

is the vector field which defines the holomorphic foliation; then the holonomy application is defined by

$$
\exp 2 \pi i X
$$

We have found two linear independent vector fields $-X, Y$ that define $h$. Therefore; they commute:

$$
[X, Y]=0 .
$$

We can describe $X$ to be so as to satisfy the commutability condition. We then show the local result:

THEOREM B. Let $p$ be a singularity of the foliation

$$
\mathcal{F}: \omega=\lambda x d y+y d x+\{\text { higher order terms }\}=0, \lambda \in \mathbb{C} .
$$


Suppose there exists a Levi foliation $\mathcal{L}$ tangent to $\mathcal{F}$. Then the singularity is normalizable in the sense of Martinet and Ramis (1982), Martinet and Ramis (1983). In particular, $\omega$ admits an analytic integrating factor.

Proof. If $\lambda \in \mathbb{C}-\mathbb{R}$, the singularity is linerizable by Poincare's Theorem. If $\lambda \in \mathbb{R}-\mathbb{Q}$, we have proved (Theorem A) that is also a linerizable singularity. Thus, we have to prove the result for $\lambda \in \mathbb{Q}$; since the singularity is a reduced one, $\lambda \in \mathbb{Q}_{+}$. Let

$$
-2 \pi i Y\left(x_{0}, y\right)=-2 \pi i \mu\left(x_{0}\right)^{k} \frac{y^{k+1}}{1-\lambda \mu\left(x_{0}\right)^{k} y^{k}} \frac{\partial}{\partial y}
$$

be the vector field whose exponential application determines the holonomy application on $x_{0}$. If there are two invariant curves through the singularity, then the vector field that defines the holomorphic distribution can be written as $x \frac{\partial}{\partial x}+y f(x, y) \frac{\partial}{\partial y}$. By solving the commutability condition $[X, Y]=0$ :

$$
\begin{aligned}
0 & =\left[x \frac{\partial}{\partial x}+y f(x, y) \frac{\partial}{\partial y}, \mu(x)^{k} \frac{y^{k+1}}{1-\lambda \mu(x)^{k} y^{k}} \frac{\partial}{\partial y}\right] \\
& =\left(y \frac{1}{\left(1-\lambda \mu(x)^{k} y^{k}\right)^{2}} d\left(\mu(x)^{k} y^{k}\right) \cdot(x, y f)-y \frac{\partial f}{\partial y} \mu(x)^{k} \frac{y^{k+1}}{1-\lambda \mu(x)^{k} y^{k}}\right) \frac{\partial}{\partial y} .
\end{aligned}
$$

Let $f(x, y)=f(x, 0)+g(x, y)$, then $f$ must be as to satisfy

$$
\left\{\begin{array}{l}
f(x, 0)=\frac{\mu^{\prime}(x) x}{\mu(x)} \\
\frac{\partial}{\partial y} \log g=k \frac{1}{y\left(1-\lambda \mu^{k} y^{k}\right)}=k \frac{\partial}{\partial y} \log \left(\frac{y}{\left(1-\lambda \mu^{k} y^{k}\right)^{\frac{1}{k}}}\right) ;
\end{array}\right.
$$

which leads us to

$$
f(x, y)=\frac{\mu^{\prime}(x) x}{\mu(x)}+\delta(x) \frac{y^{k}}{1-\lambda \mu(x)^{k} y^{k}} .
$$

The foliation on the punctured neighborhood is defined by the following 1-form

$$
\omega=x d y+y\left(\frac{\mu^{\prime}(x) x}{\mu(x)}-\delta(x) \frac{y^{k}}{1-\lambda \mu^{k} y^{k}}\right) d x
$$

or still by

$$
\begin{aligned}
\frac{\mu}{x} \omega & =\mu d y+y\left(1-\frac{\delta}{\mu^{\prime} x} \frac{y^{k} \mu^{k}}{1-\lambda \mu^{k} y^{k}}\right) d \mu \\
& =\frac{\mu^{k+1} y^{k+1}}{1-\lambda x^{k} y^{k}}\left(\frac{1-\lambda \mu^{k} y^{k}}{\mu^{k} y^{k}} \frac{d\left(\mu^{k} y^{k}\right)}{\left(\mu^{k} y^{k}\right)^{2}}+\frac{\delta}{x} d x\right) .
\end{aligned}
$$

Necessarily $\delta$ has an holomorphic extension through 0 and $\mu^{k}$ has either an holomorphic or a meromorphic extension through 0 . If it were meromorphic, the singularity would not be a reduced one, contradicting our hypotheses. The extension is then an holomorphic one. We have then a normal form for either cases: 
If $\mu^{k} \in \mathcal{O}^{*}$, we have a saddle-node; if $\mu^{k} \in \mathcal{O}-\mathcal{O}^{*}$ and let $p$ be the order of the zero of $f$ at 0 , we have a ressonant singularity.

If there is only one invariant curve through the singularity; the singularity is a saddle-node and the invariant curve is $y=0$. Therefore the vector field that defines the holomorphic distribution can be written as $X=(x+h(y)) \frac{\partial}{\partial x}+y f(x, y) \frac{\partial}{\partial y}, f(0)=0$. The holonomy is defined by the exponential application of the vector field $\frac{x}{x+h(y)} X=x \frac{\partial}{\partial x}+\frac{y f(x, y)}{x+h(y)} \frac{\partial}{\partial y}$. The commutability condition $\left[\frac{x}{x+h(y)} X, Y\right]=0$ implies that

$$
\frac{x}{x+h(y)}[X, Y]=\left(d \frac{x}{x+h(y)} . Y\right) X
$$

By solving the equation just above, we obtain that $\frac{1}{f}$ must be an holomorphic function which contradicts $f(0)=0$.

Following, we prove results that will allow us to relate the first integrals obtained on the neighborhood of each component $D_{j}$.

THEOREM C. Let $p$ be a singularity of the foliation $\mathcal{F}: \omega=0$ and

$$
\omega=f d F \text { is an holomorphic 1-form }
$$

where $F$ is a Liouvillian function and $f$ is an holomorphic integrating factor of $\omega$. There exists a Levi foliation defined by

$$
\mathcal{L}: \bar{f}(f d F)+f(\overline{f d F}) .
$$

Furthermore, if $p$ is not a linearizable ressonant singularity, then any other Levi foliation must be of the type:

$$
\mathcal{L}_{\lambda}: \lambda \bar{f}(f d F)+\bar{\lambda} f(\overline{f d F}) .
$$

Note that $\Re(\lambda F)$ is a first integral of the Levi foliation $\mathcal{L}_{\lambda}$. We can then show:

COROLlary. Let $p$ be a singularity of the holomorphic foliation $\mathcal{F}: \omega=0$. Let $F_{j}$ be Liouvillian functions and let $f_{j}$ be holomorphic functions such that

$$
\omega=f_{j} d F_{j}
$$

Suppose there exists a Levi foliation $\mathcal{L}$ tangent to $\mathcal{F}$ and suppose that $\mathfrak{R}\left(F_{1}\right), \mathfrak{R}\left(F_{2}\right)$ are first integrals of $\mathcal{L}$. Then:

$$
\frac{d F_{j}}{F_{j}}=\frac{d F_{i}}{F_{i}}
$$

Proof. Follows from $d F_{i}=\frac{f_{j}}{f_{i}} d F_{j}$ and $d\left(F_{i}+\overline{F_{i}}\right) \wedge d\left(F_{j}+\bar{F}_{j}\right)=0$.

We are then able to show: 
Theorem D. Let $\mathcal{F}$ be an holomorphic foliation and $\mathcal{L}$ be a Levi foliation tangent to $\mathcal{F}$. Suppose all singularities lie on an irredutible curve $S$; which is $\mathcal{F}$-invariant. Then $\mathcal{F}$ admits a Liouvillian first integral I defined on a neighborhood of $S$. Furthermore, $d(I+\bar{I})$ defines a Levi foliation tangent to $\mathcal{F}$.

Proof. To show the existence of a Liouvillian first integral of $\mathcal{F}$ it is enough to show the existence of a Liouvillian first integral of the reduced foliation $\mathcal{F}^{*}$. Let $D=\cup D_{j}$ be the divisor obtained on the process of reducing the singularities. Let us fix a transversal section of $\mathcal{F}^{*}$ through $D_{j}$. Since there exists a Levi foliation tangent to $\mathcal{F}^{*}$, there exists a normal coordinate system on the section so that the holonomy applications determined by the singularities on $D_{j}$ satisfy $(*)$.

For each $D_{j}$, we then find an holomorphic vector field $Z_{j}$ that defines the foliation $\mathcal{F}^{*}$ in a neighborhood of the divisor. Let $Y$ be the holomorphic vector on each transversal section which defines the holonomies. To find $Z_{j}$, all we have to do is solve the equation

$$
\left[Z_{j}, Y\right]=0 .
$$

The vector field $Z_{j}$ allows us to describe a Liouvillian first integral of the holomorphic foliation on a neighborhood of each irreduceble component $D_{j}$ of the divisor $D=\cup D_{j}$ obtained on the resolution of the singularity. Let $F_{j}$ be a Liouvillian first integral of the holomorphic foliation $\mathcal{F}^{*}$ on a neighborhood of the $D_{j}$ such that $\Re\left(F_{j}\right)$ is a first integral of $\mathcal{L}^{*}$. By Theorem $\mathrm{b}$, for each

$$
p \in D_{i} \cap D_{j}
$$

we have

$$
\frac{d F_{1}}{F_{1}}=\frac{d F_{2}}{F_{2}} .
$$

Therefore

$$
\omega^{*}=\left\{\frac{d F_{i}}{F_{i}}\right\} .
$$

is a well defined closed 1 -form. Thus

$$
I=\exp \int \omega^{*}
$$

is a Liouvillian first integral of the holomorphic foliation $\mathcal{F}^{*}$ and there is a Levi foliation $d(I+\bar{I})=$ 0 ; The Theorem is thereby proved.

\section{RESUMO}

Seja $L \subset \mathbb{C}^{2}$ uma variedade real de dimensão 3. Para todo ponto regular $p \in L$ existe uma única reta complexa $l_{p}$ no espaço tangente à $L$ em $p$. Quando o campo de linhas complexas

$$
p \mapsto l_{p}
$$

é completamente integrável, dizemos que $L$ é uma variedade de Levi. Mais geralmente, seja $L \subset M$ uma subvariedade real em uma variedade analítica complexa. Se existe uma distribuição real integrável de 
dimensão 2 em $L$ que é invariante pela estrutura holomorfa $J$ induzida pela variedade complexa $M$, dizemos que $L$ é uma variedade de Levi. Vamos provar:

Teorema. Seja $\mathcal{L}$ uma folheação de Levi e seja $\mathcal{F}$ a folheação holomorfa induzida. Então $\mathcal{F}$ tem integral primeira Liouvilliana.

Em outras palavras, se $\mathcal{L}$ é uma folheação real de dimensão 3 tal que a folheação holomorfa induzida define uma folheação holomorfa $\mathcal{F}$; isto é, se $\mathcal{L}$ é uma folheação de Levi; então $\mathcal{F}$ admite uma integral primeira Liouvilliana - uma função que pode ser construida por composição de funções rationais, exponenciações, integrações e funções racionais (Singer 1992). Por exemplo, se $f$ é uma função holomorfa e se $\theta$ é uma 1-forma real em $\mathbb{R}^{2}$; então o pull-back de $\theta$ por $f$ define uma folheação de Levi: $\mathcal{L}: f^{*} \theta=0$ a qual é tangente a folheação holomorfa $\mathcal{F}: d f=0$.

Este problema foi proposto por D. Cerveau em uma reunião (Fernandez 1997).

Palavras-chave: folheações de Levi, folheações holomorfas, singularidades, variedades de Levi.

\section{REFERENCES}

FERNANDEZ J. (editor) 1997. Ecuaciones Diferenciales: Singularidades, Universidad de Valladolid.

Martinet J \& Ramis JP. 1982. Problèmes des Modules pour des Équations Differentielles Non Linéaires du Premier Order, Math. Inst. Hautes Études Scientifiques 55: 63-124.

Martinet J \& Ramis JP. 1983. Classification Analytique des Équations Differentielles Non Linéaires Réssonantes du Premier Ordre, Ann Sc Éc Norm Sup, 16: 571-621.

Mattei JF \& Moussu R. 1980. Holonomie et Intégrales Premières, Ann Sc Norm Sup, 13.

Seindenberg A. 1968. Reduction of Singularities of The Differential Equation Ady = Bdx, Amer J Math., 248-269.

Singer M. 1992. Liouvillian First Integrals of Differential Equations, Trans American Math Soc., 333(2): 673-688. 